\title{
Políticas de Nanotecnologia em Argentina, Brasil e México: emulação e adaptação
}

Recebido: 15-06-2019

Aprovado: 25-11-2019

Noela Invernizzi, Guillermo Foladori, Tomás J. Carrozza, Edgar Záyago-Lau, Josemari Poerschke de Quevedo ${ }^{1}$

\section{Introdução}

A nanotecnologia é um campo tecnocientífico emergente, frequentemente apresentado como a base de uma nova revolução industrial (Roco, 2003), que explora as propriedades

\footnotetext{
${ }^{1}$ Noela Invernizzi. Uruguaia. Doutora em Política Científica e Tecnológica (Unicamp). Professora Associada do Setor de Educação e do PPG em Políticas Públicas da Universidade Federal do Paraná. Publicou, entre outros: "The Rise (and Fall?) of Nanotechnology Policy in Brazil"; "Nanotechnology for social needs: contributions from Latin American research in the areas of health, energy and water." e "Nanotechnology between the lab and the shop floor: what are the effects on labor?”. noela.invernizzi@ gmail.com

Guillermo Foladori. Uruguaio, Doutor em Economía (UNAM, México) e Professor Titular da Unidad de Estudios del Desarrollo, Universidad Autónoma de Zacatecas, México. Coeditou varios livros sobre nanotecnologias: Cadenas de producción de las nanotecnologías en América Latina (Bogotá, 2018), Harnessing Nanotechnology for Sustainable Development in Africa(Nairobi, 2017), Investigación y Mercado de Nanotecnologías en América Latina (México, 2016. É autor de dezenas de artigos. gfoladori@gmail.com

Tomás Javier Carrozza. Argentin. Doutorando em Desenvolvimento Econômico pela Universidade Nacional de Quilmes. Master em Agroeconomia pela Universidade Nacional de Mar del Plata, Argentina. Pesquisador da Faculdade de Ciências Agrárias da Universidade Nacional de Mar del Plata, Argentina. Autor de artigos e capítulos de livros sobre nanotecnologias e desenvolvimento sustentável, empresas de nanotecnologia na Argentina e políticas comparativas de nanotecnologia.tomascarrozza@gmail.com

Edgar Záyago-Lau. Mexicano. Doutor em Estudos do Desenvolvimento pela Universidad Autónoma de Zacatecas (UAZ), México e atualmente professor do mesmo programa onde se desempenha como Secretário Acadêmico do Doutorado em Estudos de Desenvolvimento. Co-compilador de vários livros sobre nanotecnologia, entre eles: Cadenas de producción de las nanotecnologías en América Latina (Bogotá 2018), Investigación y Mercado de Nanotecnologías en América Latina (México 2016), autor de vários artigos sobre nanotecnologia e sociedade.zayagolau@gmail.com

Josemari P. de Quevedo. Brasileira, Doutora em Políticas Públicas (Universiade Federal do Paraná, com estágio na Universidade de Coimbra); Jornalista e Mestre em Comunicação e Informação (Universidade Federal do Rio Grande do Sul). Integrante dos Grupos de Pesquisa Tecnologias Emergentes e Sociedade e Comunicação Eleitoral. Coautora do capítulo "The Rise (and Fall?) of Nanotechnology Policy in Brazil" e "Uma experiência de educomunicação inovadora: as histórias em quadrinhos sobre nanotecnologia josemari.quevedo@gmail.com
} 
específicas que a matéria exibe em escala atómica ou molecular, tipicamente abaixo dos 100 nanómetros, para elaborar novos materiais e dispositivos com aplicações em diversos setores económicos, a saúde e o ambiente (Royal Society e Royal Academy of Engineering, 2004). Trata-se de uma tecnologia com forte potencial disruptivo dos produtos e mercados existentes (Yonas e Picraux, 2000), e é considerada uma tecnologia de uso geral (enabling technology), passível de ser usada nos mais variados setores produtivos (Graham e Iacopetta, 2008).

Embora a pesquisa em nanotecnologia date da década de 1980, ocorre um salto qualitativo a partir de 2000, quando começa a ser sistematicamente estimulada por programas públicos. Em 2000, os Estados Unidos lançaram a National Nanotechnology Initiative e, na sequencia, muitos países industrializados e em desenvolvimento desenharam políticas para estimular este campo emergente. Na América Latina, ao longo da primeira década do século, 13 países a incluíram como área estratégica em suas políticas de ciência, tecnologia e inovação (PCTI). Argentina, Brasil e México, os três maiores países da região, e também os que historicamente desenvolveram as maiores capacidades científicas, ocupam as posições de liderança no desenvolvimento desta tecnologia (Foladori, Figueroa e Invernizzi, 2012).

Neste artigo examinamos, de forma comparativa, o desenho e a implementação da política de nanotecnologia em Argentina, Brasil e México, no período compreendido entre 2000 e 2016. Situamos a análise no contexto do isomorfismo resultante da emulação de políticas públicas, e evidenciamos os processos de emulação, adaptação e edição desenvolvidos em cada contexto nacional.

As dimensões examinadas se baseiam parcialmente na pesquisa desenvolvida pela OECD em 2008, Survey on Nanotechnology (OECD Working Party on Nanotechnology, 2009), para permitir a comparação com as políticas desenhadas nos países mais industrializados. $^{2}$ Estas incluem: o desenho da política; os instrumentos e o financiamento utilizados para implementá-la; os atores envolvidos na governança da nanotecnologia; as questões éticas, legais e sociais (ELS) e os aspectos ambientais, de segurança e saúde (ASS).

A informação provém de revisão da literatura e da análise de documentos de política e relatórios das agencias de promoção da ciência e tecnologia dos respectivos países.

$\mathrm{O}$ artigo se divide em cinco seções. Na primeira situamos a emergência das políticas de nanotecnologia no contexto da emulação de políticas públicas, em particular das PCTI. As quatro seções seguintes abordam as dimensões de análise da política de nanotecnologia em

\footnotetext{
${ }^{2}$ Uma nova edição deste survey foi levantada em 2016-2017. Os resultados publicados são ainda parciais.
} 
cada país: o desenho das políticas (segunda seção); os instrumentos utilizados e o financiamento alocado (terceira seção); os atores envolvidos na governança (quarta seção), e o tratamento das dimensões éticas, legais e sociais e das implicações para o ambiente, saúde e segurança (quinta seção). Encerramos o artigo com breves conclusões.

\section{A Emergência Global das Políticas de Nanotecnologia}

O isomorfismo nas políticas públicas é bastante comum. Submetidos a forças econômicas globais, e com um crescente intercâmbio de ideias resultante do desenvolvimento das comunicações, os países tendem a emular e transferir políticas. Nesse contexto, as organizações internacionais são atores relevantes que, às vezes advogam, e às vezes impõem políticas similares em países diversos (Dolowitz e Marsh, 2000).

Em cada país, instituições, políticos, funcionários públicos, grupos de pressão e a mídia são agentes que intervêm no processo de emulação de políticas (Newark, 2001). Há, ainda, a crescente influencia das Organizações Não Governamentais (ONGs), que procuram avançar suas reinvindicações em torno a problemas globais pressionando os governos para adotarem determinadas políticas (Gordenker e Weiss, 1996).

Pode ser distinguido um leque de situações na difusão de políticas. Pode haver a copia, mais ou menos intacta, ou uma emulação, que implica adoção com ajustes a diferentes circunstancias. Pode ocorrer uma hibridação de elementos de políticas tomadas de diferentes lugares. Uma categoria mais laxa seria a inspiração, quando programas desenvolvidos em outro lugar são fonte de estímulo intelectual para criar um novo programa (Rose, 1991). Como assinalam Dolowitz e Marsh (2000), enquanto a emulação de políticas é bastante frequente na fase de formulação de agendas, a combinação de elementos de diferentes modelos de referencia é mais recorrente nas fases de desenho e implementação de políticas.

Toda política emulada passa por uma adaptação às condições locais de implementação (Newark, 2001). A partir dos exemplos estrangeiros ocorrem operações de descontextualização e recontextualização mediante as quais os atores envolvidos na formulação das políticas as inscrevem num contexto local (Louvel e Hubert, 2016). Nesse sentido, Salhin e Wedlin (2008) utilizam os conceitos de tradução e edição para evidenciar que as ideias evoluem de forma diferente nos distintos contextos. 
Nas PCTI, o isomorfismo tem sido um traço característico desde sua institucionalização, em meados do século XX, abarcando suas bases conceituais, a estrutura organizacional, os instrumentos de financiamento e as formas de avaliação (Velho, 2011). Lemola (2002) afirma que a convergência das PCTI tem sido a tendência esmagadora em todos os países da OECD. As organizações internacionais tem jogado um papel importante nesse processo difundindo objetivos, racionalidades e indicadores de avaliação (Finnemore, 1993; Godin, 2009).

Nos últimos anos, salienta Velho (2011), os problemas resultantes da imitação de PCTI começaram a ser mais examinados uma vez que, não obstante os desafios globais similares, os países possuem diversas competências e capacidades, resultantes de suas diferentes historias e culturas. Elzinga e Jamison (1995) chamam a atenção para a distância entre as semelhanças na racionalidade das políticas e as diferenças em sua implementação entre países, nas ênfases colocadas, nos instrumentos usados e nas formas de gestão. Por sua vez, Dagnino e Thomas (1999) e Bagatolli (2013) atribuem a falta de resultados esperados das PCTI, especialmente quanto à inovação, à uma replicação das políticas de países desenvolvidos nos contextos muito diferentes da América Latina e do Brasil respectivamente.

O lançamento nos Estados Unidos da National Nanotechnology Initiative (NNI) pelo presidente Clinton, em 2000, (National Science and Technology Council, 2000) gerou uma onda de políticas similares não só nos países industrializados, como também em países em desenvolvimento com as mais variadas capacidades científicas. Japão, China e Coreia do Sul formularam políticas nacionais em 2001; Alemanha, Israel, e Taiwan em 2002; Reino Unido em 2003, e França em 2005 (Louvel e Hubert, 2016; Peixoto, 2013). A nanotecnologia foi designada como área prioritária no Sexto Programa Marco da Comissão Europeia, iniciado em 2002, influenciando a direção da pesquisa nesse continente e, mediante a cooperação internacional, em outros países (Pouris, Pouri e Buys, 2012). No final da década, era uma área de prioridade nacional em todos os países da OECD (Larédo, Delemarle e Kahane, 2010).

As nações em desenvolvimento se acoplaram rapidamente. De acordo com Maclurcan (2005), mais de 62 países, incluindo 25 industrializados, 18 "transicionais" e 19 em desenvolvimento tinham programas de nanotecnologia em meados da década de 2000 . Na América Latina, ao longo dessa década, 13 países incluíram a nanotecnologia como área estratégica em suas PCTI (Foladori, Figueroa e Invernizzi, 2012). Nas seções seguintes nos debruçamos sobre os casos de Argentina, Brasil e México. 


\section{As Políticas de Nanotecnologia em Argentina, Brasil e México}

A caracterização da nanotecnologia como revolução tecnológica e trampolim para a próxima revolução industrial na NNI dos Estados Unidos (National Science and Technology Council, 2000, p. 19) a colocou no quadro da forte concorrência global. Na justificativa para o que se tornaria umas das políticas científicas melhor financiadas da historia americana (Motoyama, Appelbaum e Parker, 2011), o documento sustenta que "o apoio federal à nanotecnologia é necessário para assegurar que os Estados Unidos tirem vantagens desta tecnologia estratégica e se mantenham competitivos no mercado global no futuro" (National Science and Technology Council, 2000, p. 21).

Pouco depois, a Comissão Europeia, no documento Towards a European Strategy for Nanotechnology, argumentava a necessidade de uma iniciativa europeia centralizada, com financiamento público, capaz de enfrentar os desafios intelectuais, científicos e técnicos envolvidos pelas nanociências e nanotecnologias e promover pesquisa de excelência para assegurar a competitividade europeia (European Commission, 2004, p. 11).

Neste quadro de revolução tecnológica, a emulação de políticas de nanotecnologia pelos países em desenvolvimento pode ser vista como resposta a um desafio global comum as novas condições de competitividade- tal como sugere Velho (2011), o que se verifica nos objetivos das políticas dos três países analisados. Neles, a nanotecnologia foi incluída nas PCTI com o status de área estratégica na primeira metade da década de 2000, com o objetivo de aumentar a competitividade das indústrias nacionais no mercado mundial (Ministério de Ciência e Tecnologia, 2003; República Argentina, 2005; CONACYT, 2008).

Entretanto, a transferência integral de políticas não resulta um processo simples. Não obstante as três nações se propuseram criar programas específicos para nanotecnologia, à imagem da iniciativa norte-americana e seguindo o caminho da maioria dos países da OECD -dos 24 países da OECD que responderam o survey sobre políticas de nanotecnologia, 70\% tinham formulado programas nacionais (OECD Working Party on Nanotechnology, 2009, p. 8)- apenas o Brasil conseguiu constituir um programa nacional centralizado. O plano não chegou a concretizar-se no México nem na Argentina, onde as ações se desenvolveram de forma mais dispersa.

Na Argentina, desde 2003, a Secretaria de Ciência e Tecnologia da Nação (que se tornaria Ministério de Ciência, Tecnologia e Inovação Produtiva, MCTIP, em 2007) 
estabeleceu a nanociência e a nanotecnologia como uma das temáticas prioritárias no Plano Estratégico de Ciência e Tecnologia 2005-2015 (García et al., 2012).

Em 2004 foi organizada uma reunião para avaliar o estado da pesquisa nesse campo e identificar os desafios para seu desenvolvimento. Os presentes, pesquisadores e representantes das agencias de C\&T, propuseram formar uma rede nacional de pesquisa na área e recomendaram a criação de um Programa de Vacância, para promover capacidades de P\&D e formação de recursos humanos, o que a Agência Nacional de Promoção da Ciência e da Tecnologia (ANPCYT) fez nesse mesmo ano (Andrini e Figueroa, 2008a).

Durante 2005 foi proposta a criação da Fundação Argentina de Nanotecnologia (FAN), que suscitou uma série de conflitos, incluindo denúncias públicas feitas por cientistas e jornalistas sobre o papel reservado a uma corporação estrangeira no projeto. Ao mesmo tempo, foi questionada a ingerência militar dos Estados Unidos em pesquisas de nanotecnologia no país. Esses conflitos acabaram desarticulando a ideia de elaborar um programa nacional. Posteriormente, a FAN foi reformulada como fundação sem fins de lucro com o objetivo promover a infraestrutura humana e técnica no campo da micro e nanotecnologia para fomentar sua inclusão na indústria nacional. A partir de 2007 passou a ser coordenada pelo MCTIP, afirmando sua vocação de criar vínculos entre organizações públicas que produzem $\mathrm{C} \& \mathrm{~T}$ e as empresas -especialmente pequenas e médias- que poderiam aplicar esses conhecimentos (Andrini e Figueroa, 2008b; Foladori, 2005, 2006).

O Plan Estratégico Nacional de Ciencia, Tecnología e Innovación "Bicentenario" 2006-2010 (MINCyT, 2006) situou a nanotecnologia como área estratégica, que devia ser promovida através do fomento à redes de pesquisa, formação de recursos humanos e colaboração internacional (García et al., 2012). O caráter de área estratégica foi mantido no seguinte plano de Ciência e Tecnologia Argentina Innovadora 2020 (Ministerio de Ciencia, Tecnologia e Innovación Productiva, 2012).

As atividades que desembocariam na política de nanotecnologia no Brasil se iniciaram com um workshop organizado pelo Conselho de Desenvolvimento Científico e Tecnológico (CNPq) em 2000. Os cientistas participantes concordaram na necessidade de criar um programa nacional para o campo (Knobel, 2005). Visando articular as capacidades existentes, o CNPq lançou uma convocatória de pesquisa em 2001, que redundou em quadro redes de investigação vinculando diversas instituições (Plentz e Fazzio, 2013). 
Em 2003, um grupo designado pelo Ministério de Ciência e Tecnologia (MCT) ${ }^{3}$ desenhou o Programa Nacional para o Desenvolvimento da Ciência e da Tecnologia que, no ano seguinte, seria incorporado ao Plano PluriAnual (PPA) 2004-2007 do MCT (Ministério de Ciência e Tecnologia, 2003). Em 2005, o programa foi relançado, com maior financiamento, sob o nome de Programa Nacional de Nanotecnologia, tendo maior vinculação com a política industrial, a qual considerou a nanotecnologia uma "área portadora de futuro" (Ministério de Ciência e Tecnologia, 2005). Neste plano, como nos subsequentes planos plurianuais, PPA 2007-2010 (Ministério de Ciência e Tecnologia, 2007), PPA 20122015 (Ministério de Ciência, Tecnologia e Inovação, 2012a) e PPA 2016-2019 (Ministério de Ciência, Tecnologia, Innovación e Comunicações, 2016), a nanotecnologia foi definida como área estratégica. Em 2012, foi elaborado um novo plano, a Iniciativa Brasileira de Nanotecnologia (IBN) (Ministério de Ciência, Tecnologia e Inovação, 2012b). Assim, a partir de 2004, Brasil contou com um programa de nanotecnologia centralizado, coordenado por um escritório específico no MCTI, a Coordenação Geral de Micro e Nanotecnologias, cuja direção foi exercida várias vezes por destacados pesquisadores da área (Invernizzi, Foladori e Quevedo, 2017).

No México, o Programa Especial de Ciência e Tecnologia 2001-2006 apresentou por primeira vez no país uma política de Estado em C\&T. Nesse documento a nanotecnologia foi considerada como uma área estratégica dos materiais avançados (2001). O Programa Especial de Ciência, Tecnologia e Inovação 2008-2012 voltou a situar a nanotecnologia entre as nove áreas prioritárias para o país (CONACYT, 2008).

Embora o Programa de 2001 tivesse assinalado a necessidade de contar com um programa nacional e sustentar uma rede de intercambio científico na área, o primeiro objetivo não chegou a concretizar-se, e a Rede Nacional de Nanociência e Nanotecnologia (RNYN) foi formada somente em 2010 (Red Nacional de Nanociencias y Nanotecnología, 2017). Desta forma, as ações voltadas para o fomento da nanotecnologia ocorreram de forma capilarizada em programas e ações pré-existentes no marco da política mexicana de C\&T.

O timing e os objetivos das políticas de nanotecnologia nos três países sugerem um claro processo de emulação de políticas. Neles, a nanotecnologia foi declarada área prioritária

\footnotetext{
${ }^{3}$ O Ministério de Ciência e Tecnologia mudou o nome para Ministério de Ciência, Tecnologia e Inovação (MCTI) em 2011, e para Ministério de Ciência, Tecnologia, Inovação e Comunicação (MCTIC) em 2016, o que explica os diferentes acrônimos usados nas referencias dos documentos do ministério.
} 
com a finalidade de estimular a competitividade e foi destinado financiamento público para o desenvolvimento da área.

Embora liderada pelos ministérios de C\&T e as atuantes comunidades científicas da área, a introdução da nanotecnologia nas agendas de PCTI nacionais se enquadra no ajustamento das políticas locais às recomendações que organizações internacionais vinham fazendo aos países em desenvolvimento desde a década de 1990. Estas sugeriram uma reorientação da PCTI para viabilizar a integração competitiva dos países à economia do conhecimento, o que seria obtido através da inovação e da adoção de tecnologias de ponta (OECD, 1997; OAS, 2004; World Bank, 1991). Na década seguinte, seguiram recomendações específicas da OEA para incluir a nanotecnologia como área estragégica para esse fim (OAS, 2004).

Apesar da racionalidade e dos discursos comuns, o desenho das políticas nos três países evidenciou recortes e ajustes em relação aos modelos de referencia. Enquanto no Brasil a emulação de uma política nacional de nanotecnologia centralizada foi bem sucedida, isso não ocorreu nos contextos argentino e mexicano. No primeiro, pela emergência de conflitos explícitos em torno à ingerência estrangeira na ciência local, e no México, pela mais demorada unificação dos pesquisadores em torno desse projeto. Dessa forma, as políticas de nanotecnologia se organizaram em diversos arranjos administrativos -centralizado em um caso, e capilarizado através de diversos programas nos outros dois.

\section{Instrumentos e Financiamento}

Não obstante as diferenças entre o programa centralizado no Brasil e descentralizado na Argentina e no México, os instrumentos utilizados para estimular o desenvolvimento da nanotecnologia foram semelhantes. Estes incluíram a promoção da pesquisa em redes, a formação de recursos humanos, a criação de infraestrutura, o estímulo à inovação em empresas e a cooperação internacional.

A pesquisa em rede foi o formato utilizado para vincular e ampliar a quantidade de pesquisadores no novo campo. Na Argentina, o chamado a projetos em áreas de vacância demandou a formação de redes integradas por, no mínimo, três grupos de pesquisa de diferentes províncias. Do chamado resultaram quatro redes de nanotecnologia, financiadas entre 2003 e 2007, envolvendo 200 pesquisadores (Andrini e Figueroa, 2008a). 
No Brasil, editais para formação de redes cooperativas de pesquisa em nanotecnologia, ligando cientistas de universidades e centros de pesquisa e incentivando a participação de empresas, foram lançados em 2001, 2004 e 2010, resultando em 31 redes temáticas. Em 2008, se agregaram 16 Institutos Nacionais de Ciência e Tecnologia com pesquisa na área, com formato de rede de instituições, (Plentz e Fazzio, 2013).

No México, as primeiras redes de pesquisa foram formadas espontaneamente por cientistas. Em 2006, o CONACyT lançou uma convocatória para a realização de megaprojetos de investigação científica o tecnológica em temas estratégicos, que foram implementados mediante redes temáticas. O conjunto de propostas apresentadas na área de nanotecnologia foi estruturado na Rede Temática de Nanociências e Nanotecnologia (CONACyT, 2010), que conecta 500 pesquisadores em 56 instituições (Red Nacional de Nanociencias y Nanotecnología, 2017).

A formação de novos pesquisadores em nível de pós-graduação foi estimulada para formar uma massa crítica na área. Entretanto diversas estratégias foram seguidas nos três países. Na Argentina, o conceito hegemónico entre os pesquisadores é que as ferramentas para trabalhar em nanotecnologia já existem nos currículos e disciplinas preexistentes, nas áreas de física, química e biologia. Assim, não têm sido criados cursos inteiramente focados em nanotecnologia, mas programas de especialização para complementar a formação inicial (Foladori e Carrozza, 2017). O enfoque foi totalmente diferente no México, onde proliferaram cursos especializados em nanotecnologia: em torno de 44 em nível de doutorado, 43 de mestrado e 12 cursos de graduação (Foladori et al., 2015). Brasil adotou uma perspectiva intermediária. De um lado, programas de pós-graduação existentes nas áreas de física, química, biologia, farmácia, ciência de materiais e engenharia agregaram novas linhas de pesquisa em nanotecnologia. De outro lado, foram criados cinco programas de nanotecnologia com foco em nanociências, nanomateriais, nanotecnologia farmacêutica, nanobiotecnologia e engenharia da nanotecnologia (Laureth, 2015). A diferença de enfoques resulta de sistemas de pós-graduação mais ou menos regulados, sendo que no México as universidades têm mais liberdade para formular novos cursos. Nos três países foram utilizados os programas de bolsas de pós-graduação existentes. Estas são outorgadas pelo CONICET na Argentina; pela Capes e pelo CNPq no Brasil e pelo CONACYT no México (Foladori et al., 2015; Foladori e Carrozza, 2017; Invernizzi et al., 2017). 
Esforços significativos foram realizados pelas três nações para montar uma infraestrutura de laboratórios competitiva. Brasil possui á única instalação de luz sincrotron da América Latina. Grandes laboratórios públicos foram criados ou atualizados nos últimos 10 anos e, em 2012, foi instituído o SIS-Nano, Sistema de Laboratórios de Nanotecnologia, que coordena um conjunto de grandes laboratórios estratégicos e laboratórios de diversas universidades, de caráter multiusuário, abertos também à indústria (SISNano, 2017). No México há um grande número de universidades e centros públicos de pesquisa que receberam financiamento público para montar ou atualizar laboratórios de nanotecnologia, destacando-se o Laboratório de Microscopia Central, da Universidade Nacional Autónoma de México (Foladori, Figueroa, Záyago e Invernizzi, 2012). A infraestrutura de pesquisa na Argentina é mais modesta. Não obstante, laboratórios preexistentes foram atualizados e alguns novos foram criados (Foladori, Figueroa, Záyago et al., 2012). A Fundação Argentina de Nanotecnologia, por sua vez, construiu um laboratório de uso aberto para utilização por pesquisadores e pela indústria (Foladori e Carrozza, 2017).

Deu-se bastante ênfase, nos três países, aos instrumentos para apoiar a inovação nas empresas, mediante o estímulo à pesquisa conjunta entre estas e as universidades, e através do financiamento à P\&D em empresas. Na Argentina, os Fundos Nano, criados em 2010, requerem de colaboração público-privada, entre instituições de pesquisa e empresas (ANPCT, [s.d.]), e a FAN tem um programa dirigido ao financiamento da inovação em pequenas e médias empresas de nanotecnologia e outro para a geração de startups (FAN, [s.d.]).

No Brasil, vários instrumentos de financiamento à inovação preexistentes incluiram a nanotecnologia entre as áreas focalizadas, tais como a pesquisa conjunta entre instituições de C\&T e empresas; a subvenção econômica à inovação; e o programa de bolsas para formação de recursos humanos em áreas estratégicas. Esses três programas beneficiaram, em conjunto, 122 empresas com pouco mais de R \$123 milhões entre 2003 e 2013 (Barbosa, 2017). O Sistema Brasileiro de Ciência e Tecnologia, que direciona pesquisadores para atender demandas tecnológicas de empresas, financiou três redes de inovação envolvendo 40 empresas e vários laboratórios (Ministério de Ciência, Tecnologia e Inovação, 2015).

A maioria dos fundos públicos para P\&D no México tem sido reorientados para incluir a participação do setor empresarial desde o começo da década de 2000, privilegiando a investigação aplicada (Loyola-Díaz e Paredes-López, 2009). Contudo, não foram realizados 
chamados especificamente dirigidos para nanotecnologia; as propostas na área concorreram em diversos editais de pesquisa (Casalet, 2012; Stezano, 2009).

O estabelecimento de acordos de cooperação internacional foi considerado fundamental para fortalecer a pesquisa em nanotecnologia. As três nações mantêm acordos bilaterais entre si, assim como com países da União Europeia, Estados Unidos, Japão e China. Brasil e Argentina têm, ademais, cooperação com África do Sul; Brasil com a Índia, Irã e Coreia do Sul; e México com Singapura (Ministério de Ciência e Tecnologia, 2008; Foladori et al., 2015).

Observa-se outra dimensão da emulação de políticas de nanotecnologia: o uso de instrumentos similares para o desenvolvimento científico e para a promoção da inovação nos três países, muito embora, em alguns casos, esses instrumentos estivessem vinculados à política de ciência, tecnologia e inovação de caráter mais amplo, incluindo a nanotecnologia entre outras áreas, e em outros casos, fossem explicitamente direcionados à nanotecnologia. Estes, por sua vez, são similares aos instrumentos utilizados nas políticas de nanotecnologia dos países mais industrializados elencados por Larédo et al. (2010).

Não há dados sistemáticos sobre o orçamento dedicado à nanotecnologia, sobretudo no México e na Argentina, onde não há programas centralizados. No caso do Brasil, o financiamento realizado através do Programa de Nanotecnologia não denota o conjunto do investimento, pois existem programas nos diferentes estados da federação.

Algumas estimações sugerem que Argentina investiu 50 milhões de dólares entre 2006 e 2010 (Salvarezza, 2011). A maioria do financiamento, em torno de 80\%, é público, mas algumas contrapartidas são exigidas às empresas participantes (Fischer et al., 2013). No Brasil, foram investidos em torno de 320 milhões de reais (uns 100 milhões de dólares atuais) desde a implementação do Programa Nacional de Nanotecnologia em 2004 até 2016. Entretanto, o orçamento caracterizou-se pela instabilidade, com maiores recursos entre 2004 e 2008, posterior queda, uma recuperação efêmera em 2013, e uma abrupta redução a partir de 2015 (Coordenação Geral de Micro e Nanotecnologias, 2016). No México, muitos recursos destinados a pesquisas em nanotecnologia foram implementados mediante programas gerais de financiamento à pesquisa. Alguns autores sugerem que se dedicaram 60 milhões de dólares de fundos públicos entre 2005 e 2010 (Takeuchi e Mora Ramos, 2011).

Apesar das limitações desses dados, ressalta o contraste entre o posicionamento discursivo da nanotecnologia como área estratégica para estimular a competitividade dos 
países e os orçamentos bastante restringidos e pouco sistemáticos empenhados. Ao comparar a limitação de recursos com a pluralidade de instrumentos de execução da política, ressalta uma tendência à pulverização do financiamento disponível.

\section{Atores na Governança da Nanotecnologia}

A nanotecnologia começou a se desenvolver num contexto complexo de relações ciência-sociedade, sendo objeto de forte escrutínio por diversos atores da sociedade civil. Como coloca Jasanoff (2004), desde a década de 1990 houve uma crescente mobilização de grupos sociais para reivindicar maior participação na tomada de decisões sobre ciência e tecnologia cujos resultados afetam as pessoas cotidianamente.

Em resposta a essa situação, e na esteira dos conflitos particularmente agudos em torno da biotecnologia, as políticas de nanotecnologia em muitos países industrializados incluíram ações de divulgação científica e de engajamento público que abriram espaços de participação da população. Por exemplo, a National Nanotechnology Initiative, nos Estados Unidos, vem promovendo uma serie de ações coordenadas pelo NPEC (Nanotechnology Public Engagement and Communications). ${ }^{4}$ A Comissão Europeia promoveu uma série de Diálogos sobre Nanotecnologia ${ }^{5}$ visando ampliar os atores envolvidos em sua governança. No survey levantado pela OECD, 19 dos 24 países da organização tinham desenhado mecanismos para receber input de diversos setores sociais sobre a política de nanotecnologia, e $80 \%$ dos países tinham promovido iniciativas para o envolvimento do público (OECD Working Party on Nanotechnology, 2009).

Nos três países examinados esse tipo de atividade tem sido muito limitada ou ausente. $\mathrm{Na}$ Argentina não houve nenhum tipo de consulta pública ou foro cidadão para avaliar ou comentar a política de nanotecnologia. Assim, a governança dessa tecnologia se limitou aos atores mais tradicionais no âmbito da PCTI: os cientistas e os funcionários públicos na área de ciência e tecnologia. A participação do setor empresarial foi restringida.

No caso do Brasil, em 2004, o MCT elaborou uma consulta pública via internet sobre a proposta de programa de nanotecnologia. A maioria das intervenções foi feita por cientistas, com escassíssimos aportes da indústria e da sociedade civil (Quevedo, Ferreira, e Invernizzi,

\footnotetext{
${ }^{4}$ http://www.nano.gov/npec

5 http://ec.europa.eu/nanotechnology/dialogues_en.html
} 
2016). Tanto no desenho como na implementação da política os cientistas foram o ator dominante, atuando inclusive como decisores políticos em posições-chave (Dos Santos Junior, 2011). A presença, assim como a influência, dos representantes do setor produtivo na definição da política foi pouco significativa (Mendes, 2016). Outros atores, como representantes da sociedade civil, estiveram ausentes até recentemente. A Iniciativa Brasileira de Nanotecnologia, de 2012, buscou um desenho de governança mais amplo, que incluiu o convite a representantes sindicais para participar no Comitê Interministerial de Nanotecnologia, um espaço ampliado a vários ministérios e agencias do governo criado para implementar a política (Plentz, 2016).

Um novo ator, o Partido Verde, no âmbito do Poder legislativo se conformou com a apresentação de cuatro projetos de lei sobre regulação da nanotecnologia. Os primeiros dois projetos, de 2005 e 2008, foram rapidamente arquivados. Todavia, outros dois, apresentados em 2013, geraram fortes reações da comunidade científica. Numa audiência pública realizada em 2015 no Congresso, houve um debate com participação de parlamentares, pesquisadores, um advogado e uma ONG ambientalista. Entre outros conteúdos a proposta de lei de regulação - que atualmente foi retirada para maior fundamentação- incluia a necessidade de ampliar os mecanismos de participação social (Quevedo e Invernizzi, 2016).

Seguindo os lineamentos da OECD, México criou em 2002 um mecanismo institucionalizado para receber informações públicas em qualquer tema de CT\&I. Trata-se dos Foros Consultivos de CT\&I, concebidos como órgao de expressão e comunicação dos usuários do sistema de ciência, tecnologia e inovação -cientistas e representantes do setor empresarial-, para propiciar o diálogo com os legisladores e autoridades federais e estatais. Entretanto, nada específico sobre nanotecnologia foi tratado nesses foros (FCCYT, [s.d.]).

Nos três países, a governança da nanotecnologia tem sido definida entre margens estreitas. Os principais atores envolvidos são a comunidade científica e os formuladores de política pública. Embora a participação empresarial tenha sido prevista e estimulada, esta não foi significativa em nenhum dos três países. A sociedade civil esteve quase totalmente marginalizada do processo. Esta governança limitada evidencia as dificuldades envolvidas na transferência de modelos de políticas públicas. De um lado, no contexto latino-americano a PCTI tem sido, historicamente, um terreno dominado por um ator central, a própria comunidade científica, e as agencias que a promovem. A baixa demanda por tecnologia autóctone, ou inovação, tem se traduzido em escassa participação do setor empresarial 
(Dagnino, 2014). Os objetivos dos programas de nanotecnologia, que atribuem a essa tecnologia um papel chave para a inovação e a competitividade, não contaram com o respaldo de uma participação do setor produtivo que buscasse orientar a política a seus interesses.

De outro lado, à diferença dos países mais industrializados, no contexto latinoamericano, a PCTI não tem se tornado um terreno contestado pela opinião pública - com poucas exceções, como o caso dos alimentos geneticamente modificados. Assuntos sobre ciência e tecnologia são marginais na agenda de alguns movimentos sociais, de modo que não houve grande pressão destes para abrir espaços de participação na política de nanotecnologia. Muito embora várias ONGs tenham desenvolvido ações em nível internacional para promover uma moratória da nanotecnologia, ou pressionar para que seus riscos fossem avaliados e a tecnologia fosse regulada (Lee e Kigalli, 2006; Triste, Engeman e Cruz, 2012), elas tiveram escasso impacto, inclusive no caso do México, onde atuou um braço do ETC Group, uma das ONGs mais combativas nessas pautas (ETC Group, 2017). Houve, no entanto, eventos extremos no México, com atentados de um grupo extremista contra científicos e laboratórios (Herrera Corral, 2011). No Brasil, em nível nacional, uma rede que inclui acadêmicos, funcionários de agencias de governo, sindicatos e professores, a Renanosoma, tem replicado essas pautas e mantido atividades de divulgação desde 2004. ${ }^{6}$ Alguns sindicatos da indústria química se mobilizaram nos últimos anos, demandando maior informação, transparência e participação sobre a introdução da nanotecnologia na indústria (Jensen, 2013). Na Argentina, como foi mencionado, houve certa discussão pública da nanotecnologia na mídia, quando o assunto tocou um tema sensível para o país, a ingerência militar estrangeira. No México, a seção local do IPEN (International POP Elimination Network) tem pressionado para incluir os riscos da nanotecnologia na agenda de regulação de produtos químicos (Bejarano, 2012). Em nível regional, a Rede Latino-americana Nanotecnologia e Sociedade, de caráter acadêmico, realiza pesquisas sobre o papel da nanotecnologia e suas implicações nos países em desenvolvimento, e tem realizado atividades de formação junto a ONGs e sindicatos. ${ }^{7}$ Estas atividades, contudo, não tem conseguido, por ora, influenciar de forma substancial o modelo de governança da nanotecnologia na região.

\section{Aspectos ELS (éticos, legales, sociales) e riscos}

\footnotetext{
${ }^{6} \mathrm{http}: / / \mathrm{www}$. nanotecnologiadoavesso.org/

${ }^{7}$ http://www.relans.org/
} 
As políticas de nanotecnologia, especialmente em países da Europa e nos Estados Unidos, onde manifestações sociais em torno do desenvolvimento tecnocientífico foram mais fortes, incorporaram ações para a avaliação de riscos para a saúde e o ambiente (aspectos ASS - ambiente, saúde e segurança) e dos aspectos éticos, legais e sociais (ELS) da nanotecnologia. Embora a avaliação de aspectos ASS e ELS de tecnologias emergentes não se iniciasse com as políticas de nanotecnologia, a inovação destas constituiu em propor tais avaliações desde o seu desenho inicial e durante o processo de desenvolvimento tecnológico visando, com isso, superar o modelo de avaliação ex post (Fisher, 2005; Macnaghten, Kearnes e Wynne, 2005). Ao introduzir essas dimensões, as políticas de nanotecnologia desses países desenharam uma nova forma de governança da CT\&I na qual se reconhece explicitamente as implicações sociais, éticas, e os potenciais riscos da tecnologia.

Segundo o Survey da OECD, as políticas de 18 países tinham incluído as dimensões ELS, sendo que 13 deles tinham ações e programas específicos sobre o assunto e os restantes tinham-nas incorporado como principio geral. O Survey não indagou de maneira específica sobre ações em relação aos riscos.

$\mathrm{Na}$ Argentina, em 2008, foi formado um comitê, com assessoramento da União Europeia, para elaborar um Código de Ética para a Pesquisa Responsável em Nanociência e Nanotecnologia, a semelhança do implementado na Europa em 2009 (MINCyT, 2008). A FAN realizou uma jornada sobre as implicações da nanotecnologia para a saúde, o ambiente e a sociedade em 2012, mas foi um evento sem continuidade (Balatti, 2012)

A política de nanotecnologia no Brasil mencionou escassamente os aspectos $E L S$ e não os incorporou em objetivos e metas específicos (Ministério de Ciência e Tecnologia, 2003) (Ministério de Ciência e Tecnologia, 2007). Houve avanços na IBN, que reconhece que o progresso científico e tecnológico gera implicações e riscos sociais e éticos e afirma a necessidade de maior participação social no desenvolvimento da nanotecnologia (Ministério de Ciência, Tecnologia e Inovação, 2012). Essas escassas diretrizes resultaram em ações muito limitadas. Durante o período de 2001 a 2016, houve só um chamado de investigação específico sobre as implicações sócio-econômicas e éticas da nanotecnologia, em 2004. Em 2014, o Comité Consultivo de Nanotecnologia destinou recursos para a formação de uma rede de pesquisa sobre o tema, mas nunca se concretizou. ${ }^{8}$

\footnotetext{
${ }^{8}$ Ata da reunião do CCNano de 31 de Julho de 2014.
} 
A primeira década da política de nanotecnologia no Brasil foi marcada por uma omissão quase completa da questão dos riscos. A primeira ação sistemática, o financiamento da pesquisa em nanotoxicologia, ocorreu em 2011, marcando a transição para uma abordagem mais ampla da questão na IBN de 2012, na qual a proteção do meio ambiente e dos seres humanos aparece como princípio (Ministério de Ciência, Tecnologia e Inovação, 2012, p. 74). Nos últimos anos, tanto as discussões internacionais sobre riscos e regulação, como a proposta de lei de regulação da nanotecnologia apresentada no Congresso, conduziram a maior discussão dessas temáticas.

A ausencia de referencias sobre os riscos da nanotecnología caracterizou os documentos oficiais do CONACYT, no México. Entretanto, a participação do país no Tratado de Livre Comércio de América do Norte inclui a harmonização regulatória, e algumas guias de regulação voluntária têm sido emitidas desde 2012 (Anzaldo e Herrera-Basurto, 2015; Foladori e Zayago-Lau, 2014).

México, Argentina e Brasil têm participado no Comitê de Nanotecnologia da ISO, e nos dois primeiros têm sido utilizadas as definições deste para emitir normas nacionais (Anzaldo e Herrera-Basurto, 2015; Foladori, 2017).

Observamos que a pesar do isomorfismo na racionalidade, nas metas e nos instrumentos das políticas de nanotecnologia na Argentina, Brasil e México em relação às dos países mais desenvolvidos, os aspectos sociais e a segurança dos seres humanos e do ambiente, ou o que atualmente se denomina como "desenvolvimento responsável da nanotecnologia" (Larédo et al., 2010) foi, por muitos anos, marginalizado. Essa ausência, que só começou a ser enfrentada recentemente, evidencia um processo de "edição" das políticas emuladas (Sahlin e Wedlin, 2008) e uma governança estreita da nanotecnologia. Essa situação se explica pela escassa presença de grupos sociais que reivindiquem um desenvolvimento científico seguro e ambiental e socialmente sustentável, como também responde a um forte direcionamento das ações de desenvolvimento da nanotecnologia para o mercado, que resultou numa visão do enfrentamento dos riscos e das implicações da nanotecnologia como obstáculos à promoção da inovação.

\section{Conclusões}


A análise das políticas de nanotecnologia na Argentina, Brasil e México mostra que houve um claro processo de emulação -adoção com ajustes- das politicas formuladas pelos países industrializados. A emulação se evidencia no timing de formulação das políticas, na adoção da mesma racionalidade e justificação discursiva da nanotecnologia como área estratégica para impulsar a competitividade, na identidade dos objetivos e na utilização de instrumentos semelhantes para sua implementação. Os pesquisadores do campo e os burocratas dos ministérios de ciência e tecnologia foram os atores locais relevantes nesse processo de emulação. A influencia das organizações internacionais foi também significativa, especialmente na inclusão da nanotecnologia nas PCTI dos países como área estratégica.

Para além das identidades, um exame mais apurado evidencia que ocorreram processos de adaptação e edição nas políticas locais em relação àquelas tomadas como modelo. Somente no Brasil conseguiu-se replicar o desenho de uma política nacional centralizada com orçamento específico, enquanto não se logrou nem na Argentina nem no México, e o estímulo à nanotecnologia nestes foi realizado mediante ações mais dispersas.

Muito embora os instrumentos de promoção da pesquisa, de formação de recursos humanos, de implementação de infraestrutura de laboratórios, de estímulo à interação universidade-empresa e de cooperação internacional fossem decisivos para conformar o campo nos três países, o orçamento alocado foi pequeno para uma área destacada da PCTI. Mais ainda, foi instável e pulverizado em uma multiplicidade de ações. Dessa forma, o caráter estratégico atribuído à nanotecnologia permaneceu em grande medida num plano discursivo.

A recontextualização da política de nanotecnologia nos três países mostrou dois aspectos de significativa "edição" dos modelos de referencia. Primeiramente, os mecanismos de governança mantiveram-se dentro do formato tradicional, limitando-se a três atores: cientistas, burocratas e, em muito menor medida, empresários. As inovações trazidas pelos países mais industrializados ao incluir mecanismos de participação social não tiveram eco nas formulações locais. Em segundo lugar, houve uma omissão ou inclusão parcial e tardia nas políticas locais da avaliação dos riscos e aspectos regulatórios da nanotecnologia, assim como de suas implicações éticas, legais e sociais. Os primeiros entraram em cena recentemente, de maneira ainda limitada, ao avançar a discussão sobre regulação em nível internacional.

\section{Referências}


ANDRINI, L. e FIGUEROA, S. "El impulso gubernamental a las nanociencias y nanotecnologías en Argentina." In FOLADORI, G. e INVERNIZZI, N. (Orgs.), Las nanotecnologías en América Latina. México, DF: M. A. Porrua, 2008a, pp. 33-48.

ANDRINI, L. e FIGUEROA, S. "Governmental encouragement of nanosciences and nanotechnologies in Argentina." In FOLADORI, G. e INVERNIZZI, N. (Orgs.) Nanotechnologies in Latin America, Berlin: Karl Dietz Verlag, 2008b, pp. 27-39.

ANPCT. FONTAR - Fondo Tecnológico Argentino. [s.d.], Recuperado 22 de junho de 2016, de http://www.agencia.mincyt.gob.ar/frontend/agencia/fondo/fontar

ANZALDO, M. e HERRERA-BASURTO, R. Actores, visiones y perspectivas de la gobernanza de la regulación de las nanotecnologías en México. In FOLADORI, G.; HASMY, A.; INVERNIZZI, N. e ZAYAGO LAU. E. (Orgs.). Nanotecnologías en América Latina: Trabajo y Regulación. Ciudad de México: M. A. Porrua, 2015, pp. 25-40.

BAGATtOlli, C. Política Científica e Tecnológica no Brasil: mitos e modelos num país periférico. Tese de Doutorado em Política Científica e Tecnológica. Universidade Estadual de Campinas, Campinas, 2013.

BALATTI, G. E. Primeras jornadas "Nanotecnología y sustentabilidad”. São Paulo, 25 setembro 2012. Recuperado 22 de junho de 2016, de http://factorcienciatectv.blogspot.com.br/2012/09/primeras-jornadas-nanotecnologia-y.html

BARBOSA, T. Política de Inovação em nanotecnologia no Brasil: trajetórias e empresas beneficiadas. Dissertação de Mestrado em Políticas Públicas. Universidade Federal do Paraná, Curitiba, 2017.

BEJARANO, F. "Las nanotecnologías como tema emergente en la agenda ambiental internacional y los restos de la sociedad civil en América Latina." In FOLADORI, G.; ZÁYAGO, E. e INVERNIZZI, N. (Orgs.), Perspectivas sobre el desarrollo de las nanotecnologías en América Latina. México, DF: Miguel Ángel Porrúa, 2012, pp. 235-241.

CASALET, M. "Las relaciones de colaboración entre la universidad y los sectores productivos: una oportunidad a construir en la política de innovación". In CARRRILLO, J. 
HUAlDE, A. e VILlAVICENCIO, D. (Orgs.). Dilemas de la innovación en México. Tijuana, BC: El Colegio de la Frontera Norte, 2012.

CONACYT. Programa Especial de Ciencia, Tecnología e Innovación 2008-2012. CONACYT (Consejo Nacional de Ciencia y Tecnología), 2008. Recuperado de http://www.siicyt.gob.mx/siicyt/docs/contenido/PECiTI.pdf

CONACyT (Consejo Nacional de Ciencia y Tecnologia). "Red Temática de Nanociencia y Nanotecnología". Mundo Nano, 3(2), pp. 73-100, 2010.

CONACYT (Consejo Nacional de Ciencia y Tecnología). Programa especial de ciencia y tecnología. Tomo II. CONACYT (Consejo Nacional de Ciencia y Tecnología). México DF, 2001.

CGMNT (Coordenação Geral de Micro e Nanotecnologias). Orçamento destinado a nanotecnologia. Dados consolidados por Luciana C. Estevanato. CGMNT, Ministério de Ciencia, Tecnologia e Inovação, Brasília, 2016.

DAGNINO, R. "A anomalia da política de ciência e tecnologia." Revista Brasileira de Ciências Sociais, 29(86), pp. 45-55, 2014.

DAGNINO, R. e THOMAS, H. La politica científica y tecnológica en América Latina. Redes, 12(6), pp. 49-74, 199

DOLOWITZ, D. P. e MARSH, D. "Learning from Abroad: The Role of Policy Transfer in Contemporary Policy-Making." Governance, 13(1), pp. 5-23, 2000.

DOS SANTOS JUNIOR, J. L. Ciência do Futuro: a comunidade de pesquisa e o ciclo da política de nanociência no Brasil. Tese de Doutorado em Ciências Sociais. Universidade Federal Rural do Rio de Janeiro, Rio de Janeiro, 2011.

ELZINGA, A. e JAMISON, A. (1995). "Changing policy agendas in science and technology". In Handbook of science and technology studies. Londres: Sage, 1995, pp. 572-597. 
ETC GROUP. "Nanotecnología."

2017.

Recuperado

de

http://www.etcgroup.org/es/issues/nanotechnology

EUROPEAN COMMISSION. Towards a European Strategy for Nanotechnology. European

Commission.

Brussels,

2004.

Recuperado

de

http://cordis.europa.eu/pub/nanotechnology/docs/nano_com_en_new.pdf

FAN (Fundación Argentina de Nanotecnología). Programa de Inversión en Emprendimientos. [s.d.] Recuperado 22 de junho de 2016, de http://www.fan.org.ar/acciones/programa-deinversion-en-emprendimientos/

FCCYT (Foro Consultivo de Ciencia y Tecnología). ¿Qué es el Foro Consultivo? [s.d.]. $\begin{array}{lllll}\text { Recuperado } & 2 & \text { de } & \text { fevereiro } & \text { de }\end{array}$ http://www.foroconsultivo.org.mx/home/index.php/about-foro/que-es-el-fccyt

FINNEMORE, M. "International organizations as teachers of norms: The United Nations Educational, Scientific, and Cultural Organization and science policy." International Organization, 87(4), pp. 565-597, 1993.

FISCHER, M., ROMERO, E., ZAMIT, A. L., VARELA, F., POLINO, C., e ALBERTI, J. P. Estado del Arte y Perspectivas de las Micro y Nano Tecnologías en Argentina. Asistencia Técnica para el Programa de Fortalecimiento de la Competitividad de las Pymes y Creación de Empleo en la República Argentina. Delegación de la Unión Europea en Argentina, 2013.

FISHER, E. "Lessons learned from the Ethical, Legal and Social Implications program (ELSI): Planning societal implications research for the National Nanotechnology Program." Technology in Society, 27(3), pp. 321-328, 2005.

FOLADORI, G. "Nanotecnología en Argentina. Corriendo tras la liebre." Euroresidentes.com 2005. Recuperado de https://www.euroresidentes.com/futuro/nanotecnologia/articulos/nanotecnologia-enargentina.htm

FOLADORI, G. "The U.S. Military Influence on Nanotechnology Research in Latin America | International Network of Engineers and Scientists Against Proliferation." INESAP bulletin, 
(International Network of Engineers and Scientists Against Proliferation), 2006. Recuperado de

http://www.inesap.org/bulletin-28/usmilitary\%C3\%83\%C2\%A2\%C3\%A2\%C2\%82\%C2\%AC\%C3\%A2\%C2\%84\%C2\%A2sinfluence-nanotechnology-research-latin-america

FOLADORI, G. "Occupational and environmental safety standards in nanotechnology: International Organization for Standardization, Latin America and beyond." The Economic and Labour Relations Review, 28(4), 538-554, 2017.

FOLADORI, G., ARTEAGA FIGUEROA, E., ZÁYAGO LAU, E., APPELBAUM, R., ROBLES-BELMONT, E., VILLA, L., LEOS, V. "Nanotechnology in Mexico: Key Findings Based on OECD Criteria." Minerva, 53(3), pp. 279-301, 2015.

FOLADORI, G., e CARROZZA, T. "Políticas de nanotecnología en Argentina a la luz de criterios de la OCDE." Revista Ciencia, Docencia y Tecnología, 28(55), pp. 279-301, 2017.

FOLADORI, G., FIGUEROA, S. e INVERNIZZI, N. "Nanotechnology: Distinctive Features in Latin America." Nanotechnology Law \& Business Journal, 9(1), pp. 88-103, 2012.

FOLADORI, G., FIGUEROA, S., ZÁYAGO, E., e INVERNIZZI, N. "Características distintivas del desarrollo de las nanotecnologías en América Latina." Sociologias, 14(30), pp. 330-363, 2012.

FOLADORI, G., e ZAYAGO-LAU, E. "The Regulation of Nanotechnologies in Mexico." Nanotechnology Law \& Business Journal, 11(2), pp. 164-171, 2014.

GARCÍA, M., LUGONES, M., e REISING, A. M. "Conformación y desarrollo del campo nanotecnocientífico argentino: una aproximación desde el estudio de los instrumentos de promoción científica y tecnológica." In FOLADORI, G., ZÁYAGO, E e INVERNIZZI, N. (Orgs.), Perspectivas sobre el desarrollo de las nanotecnologías en América Latina. México, DF: Miguel Ángel Porrúa, 2012.

GODIN, B. "The making of Science, Technology and Innovation Policy: Conceptual Frameworks as Narratives (1945-2005)." Centre Urbanisation, Culture, Societé, Institut National de la Récherche Scientifique, Montréal, 2009. 
GORDENKER, L. e WEISS, T. "Pluralising global governance: Analytical approaches and dimensions." In NGOs, the UN, and Global Governance. Boulder: Westview, 1996, pp. 1327.

GRAHAM, S. e IACOPETTA, M. "Nanotechnology and the Emergence of a General Purpose Technology." Annals of Economics and Statistics N. 115/116. Recuperado de http://ssrn.com/abstract $=1334376$

HERRERA CORRAL, G. "Stand up against the anti-technology terrorists." Nature, 476(7361), pp 373-373, 2011.

INVERNIZZI, N., FOLADORI, G., e QUEVEDO, J. (2017). "The Rise (and Fall?) of Nanotechnology Policy in Brazil." In BOWMAN, D. et al. (Org.), The Politics and Possibilities of Emerging Technologies. Berlin: IOS Press, 2017, pp. 69-90,

JASANOFF, S. "Science and citizenship: a new synergy." Science and Public Policy, 31(2), pp. 90-94, 2004.

JENSEN, T. "Nanotecnologias na experiência recente de negociação coletiva do movimento sindical brasileiro." Apresentado em $1^{\circ}$. Seminário Internacional Nanotecnologia e Trabalho, Curitiba, 3. set. 2013.

KNOBEL, M. "O futuro da nanotecnologia no Brasil. Vinte anos não são nada?"Ciência $e$ Cultura, 57(1), pp. 4-5, 2005.

LARÉDO, P., DELEMARLE, A. e KAHANE, B. "Dynamics of Nanosciences and Technologies: Policy Implication." STI Policy Review, 1(1), pp. 43-62, 2010.

LAURETH, W. "Formación de la fuerza de trabajo en nanotecnología en Brasil: recomendaciones de las políticas, acciones de las universidades y de las empresas." In FOLADORI, G.; HASMY, A.; INVERNIZZI, N. e ZAYAGO LAU. E. (Orgs.). Nanotecnologías en América Latina: Trabajo y Regulación. Ciudad de México: M. A. Porrua, 2015, pp. 171-188. 
LEE, J., e KIGALLI, R. Global Nanotechnology Advocacy by NGOs. Suíça: Centre for Applied Studies in International Negotiations, 2006.

LEMOLA, T. Convergence of national science and technology policies: the case of Finland. Research Policy, (31), pp. 1481-1490, 2002.

LOUVEL, S., e HUBERT, M. L'usage des exemples étrangers dans les politiques de financement de la recherche. Les nanosciences et nanotechnologies en France. Revue française de sociologie, 57(3), pp. 473-501, 2016.

LOYOLA-DÍAZ, e PAREDES-LÓPEZ, O. La ciencia y la innovación en México, en la encrucijada. La Crónica de Hoy. 4. Feb. 2009. Recuperado de: http://www.comunicacion.amc.edu.mx/amc-en-medios/la-ciencia-y-la-innovacion-en-mexicoen-la-encrucijada

MACLURCAN, D. "Nanotechnology and Developing Countries - Part 2: What Realities?" Azojono. Online Journal of Materials, 2005. Recuperado de https://www.azonano.com/article.aspx?ArticleID=1429

MACNAGHTEN, P., KEARNES, M. B., e WYNNE, B. Nanotechnology, Governance, and Public Deliberation: What Role for the Social Sciences? Science Communication, 27(2), pp. 268-291, 2005.

MCT (Ministério de Ciência e Tecnologia). Relatório analítico Programa de C,T\&I para Nanotecnologia. Coordenação Geral de Micro e Nanotecnologias. Coordenação Geral de Micro e Nanotecnologia, Brasília, Ministério de Ciência e Tecnologia, 2008.

MCT (Ministério de Ciência e Tecnologia). Programa de desenvolvimento da nanociência e da nanotecnologia. Brasília, Ministério da Ciencia e Tecnologia, 2003.

MCT (Ministério de Ciência e Tecnologia). Programa Nacional de Nanotecnologia. Coordenação Geral de Micro e Nanotecnologia, Brasília, Ministério de Ciência e Tecnologia, 2005.

http://www.mct.gov.br/index.php/content/view/27137/Programa_Nacional_de_Nanotecnologi a.html 
MCT (Ministério de Ciência e Tecnologia). Ciência, Tecnologia e Inovação para o Desenvolvimento Nacional. Plano de Ação 2007-2010. Brasília, Ministério da Ciência e Tecnologia, 2007.

MCTI (Ministério de Ciência, Tecnologia e Inovação). Iniciativa Brasileira de Nanotecnologia. Coordenação Geral de Micro e Nanotecnologia, Brasília, Ministério de Ciência, Tecnologia e Inovação, 2012a.

MCTI (Ministério de Ciência, Tecnologia e Inovação). Estratégia Nacional de Ciência, Tecnologia e Inovação 2012-2015. Brasília, Ministério da Ciência, Tecnologia e Inovação, $2012 b$.

MCTI (Ministério de Ciência, Tecnologia e Inovação). Relatório de Gestão, Exercício 2014. Coordenação Geral de Micro e Nanotecnologia, Brasília, Ministério de Ciência, Tecnologia e Inovação, 2015.

MCTIC (Ministério de Ciência,Tecnologia, Innovación e Comunicações). Estratégia Nacional de Ciência, Tecnologia e Inovação 2016-2019. Brasília, Ministério da Ciência, Tecnolgoia, Inovação e Comunicações, 2016.

MENDES, A. A política de nanotecnologia no Brasil. Entrevista, Brasília, 6 julho 2016.

MINCyT (Ministerio de Ciencia y Tecnología). Plan estratégico nacional de ciencia, tecnología e innovación "Bicentenario" (2006-2010). Buenos Aires, Ministerio de Ciencia, Tecnología e Innovación Productiva, 2006. Recuperado de http://www.mincyt.gob.ar/planesnacionales/plan-estrategico-nacional-de-ciencia-tecnologia-e-innovacion-bicentenario-20062010-8017

MINCyT (Ministerio de Ciencia y Tecnología). Avanza la creación de un código de ética para la investigación en nanotecnología, 2008. Recuperado 17 de junho de 2016, de http://www.mincyt.gob.ar/noticias/avanza-la-creacion-de-un-codigo-de-etica-para-lainvestigacion-en-nanotecnologia-4206

MINCyT (Ministerio de Ciencia, Tecnología e Innovación Productiva). Argentina innovadora 2020. Plan Nacional de Ciencia, Tecnología e Innovación. Lineamientos estratégicos 2012- 
2015. Buenos Aires, MINCYT, 2012. Recuperado de http://www.mincyt.gob.ar/adjuntos/archivos/000/022/0000022576.pdf

MOTOYAMA, Y., APPELBAUM, R. e PARKER, R. (2011). "The National Nanotechnology Initiative: Federal support for science and technology, or hidden industrial policy?" Technology in Society, 33(1-2), pp. 109-118, 2011.

NSTC (National Science and Technology Council). National Nanotechnology Initiative. The initiative and its implementation plan. Washington DC, National Science and Technology Council. Recuperado de http://www.wtec.org/loyola/nano/IWGN.Implementation.Plan/nni.implementation.plan.pdf

NEWARK, A. J. An integrated approach to policy transfer and diffusion. The Rewiew of Policy Research, 19 (2), pp. 151-178, 2002.

OECD (Organization for Economic Development). National Innovation Systems. Paris: OECD, 1997.

OECD (Organization for Economic Development). Working Party on Nanotechnology. Inventory of National Science, Technology and Innovation Policies for Nanotechnology 2008 (p. 1-95). Paris: Organization for Economic Cooperation and Development, 2009. Recuperado de http://www.oecd.org/sti/nano/43348394.pdf

OAS (Organization of American States). Final report of the fourth regular meeting of the inter-american committee on science and technology (COMCYT). Inter-American Committee on Science and Technology. Washington DC, Organization of American States, 2004. Recuperado de http://portal.oas.org/LinkClick.aspx?fileticket=yKkiwQONYYk\%3D\&tabid=1601

PEIXOTO, F. J. M. Nanotecnologia e sistemas de inovação: implicações para política de inovação no Brasil. Tese de Doutorado em Economia. Universidade Federal do Rio de Janeiro, Rio de Janeiro, 2013.

PLENTZ, F. A política de nanotecnologia no Brasil, Entrevista, Belo Horizonte, 6 outubro 2016. 
PLENTZ, F., e FAZZIO, A. Considerações sobre o Programa Brasileiro de Nanotecnologia. Ciência e Cultura, 65(3), pp. 23-27, 2013.

POURIS, A., POURI, A., e BUYS, A. "Nanotechnology and biotechnology research in South Africa: Technology management lessons from a developing country." Apresentado em Technology Management for Emerging Technologies, Vancouver, Canada, 23 julho 2012. Recuperado de http://ieeexplore.ieee.org/document/6304055/?reload=true

QUEVEDO, J., FERREIRA, J. H. e INVERNIZZI, N. "O mini-público como modelo comunicacional de promoção ao debate sobre riscos da nanotecnologia no Brasil." Apresentado em XVII Congresso de Ciências da Comunicação na Região Sul, Curitiba, 28 Maio 2016.

QUEVEDO, J. e INVERNIZZI, N. "A rede de atores nas proposições para a regulação da nanotecnologia no Brasil."Apresentado em XI Jornadas Latinoamericanas dos Estudos Sociais da Ciência e da Tecnologia, Curitiba, 25 julho 2016.

REDNyN (Red Nacional de Nanociencias y Nanotecnología). La Red Nacional de Nanociencias y Nanotecnologías, México DF, 2017. Recuperado 16 de setembro de 2017, de http://www.nanored.org.mx/objetivos.aspx

REPÚBLICA ARGENTINA. Decreto Presidencial 380/2005. Autorízase al Ministerio de Economía y Producción a constituir la Fundación Argentina de Nanotecnología. Buenos Aires, Presidencia de la República Argentina, 2005.

ROCO, M. C. "Broader societal issues of nanotechnology." Journal of Nanoparticle Research, 5, pp. 181-189, 2003.

ROSE, R. What is Lesson-Drawing? Journal of Public Policy, 11(01), pp. 3-30, 1991.

RS\&RAE (Royal Society \& Royal Academy of Engineering). Nanoscience and nanotechnologies: opportunities and uncertainties. London, The Royal Society; Royal Academy of Engineering, 2004. 
SAHLIN, K., e WEDLIN, L. "Circulating Ideas: Imitation, Translation and Editing." In The Sage Handbook of Organizational Institutionalism. Los Angeles: Sage, 2008, pp. 218-242.

SALVAREZZA, R. Situación de la difusión de la nanociencia y la nanotecnología en Argentina. Mundo Nano, 4(2), pp. 18-21, 2011.

SISNANO. Sistema Nacional de Laboratórios em Nanotecnologia - SIS-Nano. Brasília, Ministério de Ciência, Tecnologia e Inovação, 2014. Recuperado de http://www.mcti.gov.br/SisNano

STEZANO, F. El rol de los programas en CyT y organizaciones intermedias en las relaciones ciencia-industria. Revisión analítica y experiencias en México. In Martínez, A.M.; LÓPEZ DE ALBA, P., GARCÍA, A. e ESTRADA, S. (Orgs.), Innovación y competitividad en la sociedad del conocimiento. México, D.F: Plaza y Valdés, 2009, pp. 483-508.

TAKEUCHI, N. e MORA RAMOS, M. E. Divulgación y formación en nanotecnología en México. Mundo Nano, 4(2), pp. 59-64, 2011.

TRISTE, E., ENGEMAN, C. e CRUZ, H. "Nano Regulatory Policy and NGOs: a global view." Apresentado em Anual Meeting of the Society for the Study of Nanotechnology, University of Twente, Netherlands, 25 Outubro 2012.

VELHO, L. Conceitos de Ciência e a Política Científica, Tecnológica e de Inovação. Sociologías, 13(26), pp. 128-153, 2011.

World Bank. World Development Report 1991: The Challenge of Development. New York: Oxford University Press, 1991.

YONAS, G. e PICRAUX, S. T. "National needs drivers for nanotechnology". In ROCO, M. e W. S. BAINBRIDGE, W.S. (Orgs.), Societal Implications of Nanoscience and Nanotechnology. Arlington, VA: National Science Foundation, 2000, pp 37-44.

\section{Políticas de Nanotecnologia em Argentina, Brasil e México: emulação e adaptação}




\title{
Resumo
}

O objetivo deste artigo é examinar, de forma comparativa, o desenho e a implementação das políticas de nanotecnologia na Argentina, Brasil e México, de 2000 a 2016. A análise situa-se no contexto da recorrente emulação de políticas de ciência, tecnologia e inovação, e examina as adaptações realizadas durante a implementação. As dimensões analisadas se baseiam no Survey sobre Nanotecnologia, realizado em 2008 pela OECD, para facilitar a comparação internacional. Estas incluem: desenho da política, instrumentos e financiamento, atores envolvidos na governança, avaliação de questões sociais, éticas e riscos. A pesquisa foi desenvolvida mediante revisão de literatura e análise documental. Os resultados evidenciam a emulação da racionalidade e dos instrumentos das políticas dos países industrializados. Porém, a forma de governança e a avaliação das implicações sociais e dos riscos da nanotecnologia foram aspectos não emulados evidenciando processos de edição local nos três países latinoamericanos.

Palavras-chave: políticas de nanotecnologia, emulação de políticas públicas, instrumentos de política, governança.

\section{Nanotechnology Policies in Argentina, Brazil and Mexico: emulation and adaptation}

\begin{abstract}
The purpose of this article is to examine, in a comparative manner, the design and implementation of nanotechnology policy in Argentina, Brazil and Mexico, from 2000 to 2016. The analysis is placed in the context of recurrent emulation of science, technology and innovation policy and examines the adaptations carried out during the implementation phase. The dimensions analyzed are based on the 2008 OECD Survey on Nanotechnology in order to facilitate international comparison. They include: policy design; instruments and funding; actors engaged in governance; ethical, social and risks assessment. The research was carried out through literature review and documental analysis. Main results show the emulation of the rationale and instruments used by industrialized countries' policies. However, the governance approach and the assessment of social implications and risks were not emulated, evidencing local editing processes in the three Latin-American countries.
\end{abstract}

Key-words: nanotechnology policy; public policy emulation, policy instruments, governance.

\section{Políticas de Nanotecnología en Argentina, Brasil y México: emulación y adaptación}

\section{Resumen}

El objetivo de este artículo es examinar, de forma comparativa, el diseño y la implementación de las políticas de nanotecnología en Argentina, Brasil y México, de 2000 a 2016. El análisis se sitúa em el contexto de la recurrente emulación de políticas de ciencia, tecnología e innovación, y examina las adaptaciones realizadas durante la implementación. Las dimensiones analizadas se basan en el Survey sobre Nanotecnología, realizado en 2008 por la OECD, para facilitar la comparación a nivel internacional. Estas incluyen: diseño de la política, instrumentos y financiamiento, actores involucrados en la gobernanza, evaluación de cuestiones sociales, éticas y riesgos. La investigación fue desarrollada mediante revisión de literatura y análisis documental. Los resultados evidencian la emulación de la racionalidad y de los instrumentos de las políticas de los países industrializados. Sin embargo, la forma de gobernanza, y la evaluación de las implicaciones sociales y los riesgos de la nanotecnología fueron aspectos no emulados, mostrando procesos de edición local de las políticas en los tres países latinoamericanos.

Palabras clave: políticas de nanotecnología, emulación de políticas públicas, instrumentos de política, gobernanza. 